\title{
Signal Quality Monitoring Algorithm Applied to Galileo Signals for Large Evil Waveform Threat Space
}

\author{
Ikhlas Selmi, Paul Thevenon, Christophe Macabiau, ENAC, Université de Toulouse \\ Olivier Julien, $u$-Blox $A G$, Switzeland \\ Mikael Mabilleau, $G S A$
}

\section{BIOGRAPHY}

Ikhlas Selmi is currently part of the SIGnal processing and NAVigation (SIGNAV) research axis of TELECOM team at ENAC. She graduated as engineer in Telecommunication and Signal Processing from ESIEE. She received her Ph.D. in 2013 from Telecom SudParis on mitigating GNSS interference between indoor and outdoor signals. She is currently working on GNSS signal distortion generated by the satellite payload.

Paul Thevenon graduated as electronic engineer from Ecole Centrale de Lille in 2004 and obtained in 2007 a research master at ISAE in space telecommunications. In 2010, he obtained a PhD degree in the signal processing laboratory of ENAC in Toulouse, France. From 2010 to 2013, he was employed by CNES, the French space agency, to supervise GNSS research activities and measurement campaigns. Since the July 2013, he is employed by ENAC as Assistant Professor. His current activities are GNSS signal processing, GNSS integrity monitoring and hybridization of GNSS with other sensors.

Christophe MACABIAU graduated as electronics engineer in 1992 from the ENAC in Toulouse, France. Since 1994, he has been working on the application of satellite navigation techniques to civil aviation. He received his Ph.D in 1997 and has been in charge of the signal processing lab of ENAC from 2000 to 2012. He is currently the head of the TELECOM team of ENAC.

Olivier Julien is a Senior Principal Engineer in u-blox AG, Switzerland since December 2018. He was the head of the Signal Processing and Navigation (SIGNAV) research group of the TELECOM laboratory of ENAC, in Toulouse, France. He received his engineer degree in 2001 in digital communications from ENAC and his PhD in 2005 from the Department of Geomatics Engineering of the University of Calgary, Canada. His research interests are turned towards the use of satellite-based navigation systems for safe navigation.

Mikael Mabilleau is a standardisation engineer on SBAS in the EGNOS exploitation team of the European GNSS Agency (GSA). Since his graduation as engineer from the French civil aviation school (ENAC) in 2006, he has been involved in GNSS standardization activity carried by the main civil aviation standardisation bodies such as the EUROCAE WG 62 on Galileo, RTCA Special Committee 159 on GPS and the International Civil Aviation Organisation (ICAO) Navigation System Panel in charge of ICAO standard. Mikael is involved in the evolution of GNSS concepts (ARAIM and SBAS L1L5) supporting the development of the associated standards (SARPs and MOPS) for use in aviation.

\footnotetext{
ABSTRACT

After the observed Evil Wave Form (EWF) event in 1993, an ICAO Threat Model (TM) and Threat Space (TS) are proposed for GPS L1 C/A signal to characterize those distortions [1]. Then, a Signal Quality Monitoring (SQM) algorithm is designed to protect civil aviation users from the potential risk of these signal anomalies. Under the development of new ICAO standards for Galileo, the EWF for Galileo signals have to be characterized in order to design suitable SQM algorithm to protect aviation user when using those new signals in operation. Based on the ICAO TM and the TS adapted to Galileo signals, a SQM design needs to be defined for Galileo E1 and E5 signals in Dual Frequency Multi-Constellation (DFMC) systems. This paper focuses on the SQM design and compliance test when considering a very large EWF TS including the Galileo TS. The hazardous EWF cases that need to be detected by the SQM are those characterized by a differential bias larger than the Maximum tolerable Error (MERR) within the tested TS. The EWF differential bias is defined as the worst bias observed when the anomaly occurs on the satellite after (called rising scenario) or before (called risen scenario) it is being monitored by the SBAS reference stations. The required missed detection and false alarm probabilities for the tested TS are evaluated based on the called time-varying MERR methodology [2] and SBAS parameters. The
} 
paper proposes a SQM and code pseudorange jump monitor (CCI) that is compliant with the SBAS integrity and continuity requirements considering the TS for Galileo signals.

\section{INTRODUCTION}

In 1993, the first important GNSS signal distortion due to a payload failure was observed. Other cases [3] occurred recently confirming the potential risk of this type of distorted signals. To characterize those distortions and protect civil aviation users from their potential risk, three threat characterizations, also known as Threat Models (TM), were adopted in 2006 by ICAO for GPS L1 C/A [1]. They are classified in three categories: TM-A, TM-B and TM-C. TM-A is associated with a failure in the Navigation Data Unit (NDU). It appears on the C/A code as a leading or lagging falling edge on all the positive chips with respect to their expected end-time. TM-B models degradation in the analog section of the satellites by an amplitude modulation applied to the correct signal. It can be also seen as the output of a second order system taking the nominal C/A code as an input. TM-C is a combination of both digital and analog failures. The proposed TM for GPS L1 C/A signal is here assumed to be also valid for new GNSS signals as the Galileo E1c and E5a ones.

The GPS L1 C/A ICAO Threat space for EWF TM is defined in Table 1. In this paper, the main objective is to propose a robust Signal Quality algorithm to protect SBAS user against any EWF event with possible integrity risk for SBAS considering the Threat Space given in Table 2.

Table 1. Standardized Threat Space for GPS L1 C/A [1]

\begin{tabular}{|c|c|c|c|}
\hline & $\Delta(\mu s)$ & $\sigma($ Mnepers/s) & $f_{d}(\mathrm{MHz})$ \\
\hline TM-A & $\begin{array}{c}{[-0.12 ; 0.12] \text { chip or }} \\
{[-117.3 ; 117.3] \mathrm{ns}}\end{array}$ & - & - \\
\hline TM-B & - & {$[0.8 ; 8.8]$} & {$[4 ; 17]$} \\
\hline TM-C & $\begin{array}{c}{[-0.12 ; 0.12] \text { chip or }} \\
{[-117.3 ; 117.3] \mathrm{ns}}\end{array}$ & {$[0.8 ; 8.8]$} & {$[7.3 ; 13]$} \\
\hline
\end{tabular}

Table 2. Tested Threat Space for Galileo E1c and E5a

\begin{tabular}{|c|c|c|c|c|}
\hline \multicolumn{2}{|c|}{ ICAO parameters } & $\Delta(\mu s)$ & $\sigma$ (Mnepers/s) & $f_{d}(\mathrm{MHz})$ \\
\hline \multirow{2}{*}{ TM-A } & Galileo E1c & {$[-0.16 ; 0.16]$} & \multirow[t]{2}{*}{ ( } & \multirow[t]{2}{*}{ 然 } \\
\hline & Galileo E5a & {$[-0.16 ; 0.16]$} & & \\
\hline \multirow{2}{*}{ TM-B } & Galileo E1c & - & {$[0.1 ; 700]$} & {$[0.1 ; 55]$} \\
\hline & Galileo E5a & - & {$[0.1 ; 370]$} & {$[0.1 ; 30]$} \\
\hline \multirow{2}{*}{ TM-C } & Galileo E1c & {$[-0.16 ; 0.16]$} & {$[0.1 ; 700]$} & {$[0.1 ; 55]$} \\
\hline & Galileo E5a & {$[-0.16 ; 0.16]$} & {$[0.1 ; 370]$} & {$[0.1 ; 30]$} \\
\hline
\end{tabular}

For EWF detection purpose, SBAS uses a general monitor named the Code Carrier Incoherency (CCI) monitor which is applied on the reference station code and carrier measurements. It also uses the Signal Quality Monitor (SQM) algorithm designed to cover the hazardous cases in the tested Galileo TS. The CCI and SQM monitors have to be compliant with the allocated integrity risk requirements in terms of false alarm and missed detection probabilities. The goal of the SBAS monitoring is that any EWF with a bias exceeding the Maximum tolerable Error Range (MERR) determined for each signal is detected to trigger an alarm and alert the user. In this study, an SQM called SQM2b and the CCI are presented and their performances are assessed with respect to the considered MERR limit.

\section{EVIL WAVEFORM DIFFERENTIAL BIAS AND MAXIMUM TOLERABLE ERROR RANGE (MERR)}

\section{Evil Waveform biases evaluation}

Depending on the moment when the EWF occurs on the satellite, two scenarios can be distinguished before (rising scenario) or after (risen scenario) the satellite is being visible to several SBAS reference stations. 
In the rising scenario, the EWF event occurs when the affected satellite is not visible from the SBAS network. The affected satellite only becomes visible from the SBAS network later. In this case:

- Since the user and the reference station are both immediately affected by an EWF-induced "steady state" differential bias. The following equation gives the differential bias estimation in time, considering that the EWF-induced bias creates a transient error due to the smoothing filter of the user and reference receivers:

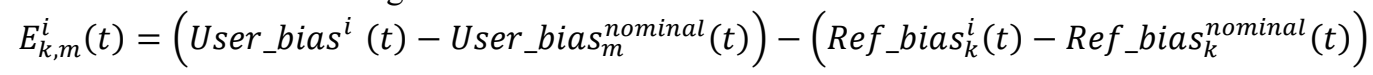

where

- User_bias and Ref_bias are respectively the user and Reference station pseudorange measurements

- $i$ denotes the $i$-th distortion of the threat space

- $m$ represents the $m$-th type of user of the user design space (defined in Table 3 )

$\circ \quad k$ represents the $k$-th type of reference receiver of the reference receiver design space (defined in Table 3 )

If at $t=t_{s s}$, the user and reference smoothing filter come to the end of their transient period, the "steady state" differential bias observed in the rising case is deduced as:

$$
\text { Max_Diff_Bias } \text { rising }_{-}^{i}=\operatorname{abs}\left(\max _{k, m}\left(E_{k, m}^{i}\left(t_{s s}\right)\right)\right)
$$

- The SBAS reference stations will not see a jump in their pseudorange measurements since the satellite already has undergone the EWF event. In this case, the bias on the pseudorange might be interpreted as a bias in the satellite clock.

The so-called risen case appears when the signal is undergoing an EWF when being visible to several SBAS monitor stations. So, the satellite is already monitored by the considered reference stations.

- In this case the user and the reference station will experience absolute biases that will vary differently during the transient phase of their smoothing filters depending on the filter time constant as shown in the example of Figure 1. Hence the differential error that needs to be estimated to evaluate the worst effect of an EWF is the maximum differential bias during the transition phase. Note that this error may be larger than the steady state error if the filter time constant of the user and reference smoothing filter are different. It is computed as:

$$
\text { Max_Diff_Bias } s_{\text {risen }}^{i}=\operatorname{abs}\left(\max _{\substack{k, m \\ t}}\left(E_{k, m}^{i}(t)\right)\right)
$$

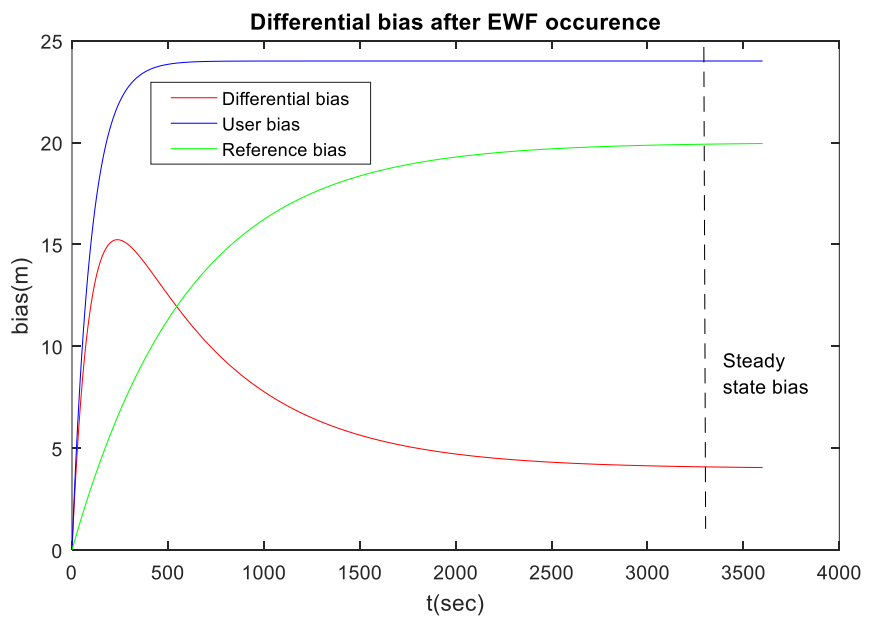

Figure 1. Example of time evolution of user, reference and differential biases with $100 \mathrm{~s}$ and $600 \mathrm{~s}$ on airborne and ground smoothing filter respectively.

- In the risen case, the Code-Carrier Incoherence (CCI) monitor can be used to detect the jump on the reference station measurements, since the reference station is monitoring the satellite before and after the EWF occurrence. 


\section{MERR limit}

The SBAS authorized limit on the pseudorange measurement bias is called the Maximum tolerable ERror Range (MERR). To estimate it for DFMC SBAS, a simple MERR formula is provided for WAAS (single frequency) [4] as:

$$
M E R R=5.33 \sqrt{\sigma_{U D R E}{ }^{2}+\left(F_{p p} \sigma_{U I V E}\right)^{2}}
$$

Where $\sigma_{U D R E}$ and $\sigma_{U I V E}$ are respectively the standard deviation of the UDRE (User Differential Range Error) and the GIVE (Grid Ionosphere Vertical Error) monitors, and $F_{p p}$ is the obliquity factor. Since the ionospheric term can be removed for dual frequency SBAS system, the MERR becomes:

$$
M E R R=5.33 \sigma_{D F R E}
$$

Where $\sigma_{D F R E}$ is the standard deviation of the Dual-frequency Range Error (DFRE).

Assuming that SBAS's minimum targeted DFRE is 4, the MERR limit is $3.64 \mathrm{~m}$ in dual frequency case. The resulting MERR limit on $\mathrm{E} 1$ and $\mathrm{E} 5 \mathrm{a}$ are given based on the differential bias formula for iono-free dual frequency measurements:

$$
\Delta b_{E W F, D F}=\left|2.26 \Delta b_{E W F, L 1}-1.26 \Delta b_{E W F, L 5}\right|
$$

Where $\Delta b_{E W F, D F}$ is the dual frequency bias. $\Delta b_{E W F, L 1}$ and $\Delta b_{E W F, L 5}$ are respectively the E1 and E5a EWF biases.

Assuming that an EWF can only occur on one signal carrier at a time, the MERR limit for E1 and E5a are respectively $\Delta b_{E W F, L 1}=$ $\Delta b_{E W F, D F} / 2.26=1.61 \mathrm{~m}$ and $\Delta b_{E W F, L 5}=\Delta b_{E W F, D F} / 1.26=2.98 \mathrm{~m}$. In order to be more conservative, an extra margin is added on the single-frequency MERR by setting it to $1 \mathrm{~m}$ and $2 \mathrm{~m}$ for E1 and E5a respectively.

A more exhaustive approach is proposed in [2] to define a more generic MERR, which is distortion-dependent and which considers the impact of the distortion on both the range domain and the monitor sensitivity. The obtained MERR values with this approach (while taking into account the SBAS constraints for DFMC with a VAL limit of 35m) should be larger than the $1 \mathrm{~m}$ and $2 \mathrm{~m}$ estimated based on the static MERR. Hence, the $1 \mathrm{~m}$ and $2 \mathrm{~m}$ values for E1 and E5a are considered enough conservative to be used as the tolerable threshold on the evaluated differential biases for risen and rising cases.

\section{EVIL WAVEFORM DETECTORS DEFINITION}

\section{SBAS Monitors}

Two types of monitors are considered here: the CCI and the SQM2b. The definition of each monitor test is given in this section.

\section{CCI monitor}

The calibrated CCI detector is an additional barrier on the reference bias. It is able to detect a jump on the code measurement when observing its time variation. This detector is only efficient in the risen satellite situation since the time evolution of the code bias induced by an EWF can only be observed if the satellite is monitored when EWF occurs. In the Rising case, the CCI monitor is not applied.

In this simulator developed by ENAC, the CCI test Test ${ }_{C C I}$ is computed as the code bias given by the E-L measurements for the distorted (bias $S_{C C I}^{E W F}$ ) and nominal (bias ${ }_{C C I}^{\text {nominal }}$ ) correlation function. The CCI metric threshold threshold ${ }_{C C I}$ is proportional to the CCI standard deviation $\sigma_{C C I}$. The CCI test is given by the following expression:

$$
\text { Test }_{C C I}=\frac{\text { bias }_{C C I}^{E W F}-\text { bias }_{C C I}^{\text {nominal }}}{\text { threshold }_{C C I}}
$$

In practice, the difference between an instantaneous iono-free code-minus-carrier observation (used as an observation of bias ${ }_{C C I}^{E W F}$ ) and the average over the past values of this same iono-free code-minus-carrier (considered as bias nominal) is currently being used in SBAS. The $\sigma_{C C I}$ used in the simulator corresponds to this definition and is mainly affected by the code measurement noise.

Different CCI formula which is also called CCD as Code Carrier Divergence (for GBAS) can be proposed [5]. It is based on the use of a time derivative of the code minus carrier measurements. This formula would improve the performance of the CCI (if needed).

The implemented CCI monitor for this study is a simplified model (using the Test ${ }_{C C I}$ expression) of the real detector. It assumes that the EWF effect on the carrier component is too low and can be ignored. This model is representative of the processing methods used in the SBAS CCI or GBAS CCD monitors.

\section{$\underline{\text { SQM2b metrics }}$}


The SQM2b is based on the proposed metric in [6], [7] and on the observation of the cross-correlation function between the incoming (distorted) signal and the local replica computed in the receiver as shown in Figure 2. It uses three types of correlator output combinations computed as the simple ratio $\left(M_{s r}\right)$, the symmetric difference $\left(M_{s d r}\right)$ and the double difference ratio $\left(M_{d d r}\right)$ metrics. These metrics are defined as follows:

where

$$
M_{s r}=\frac{I_{x}}{I_{0}}, M_{s d r}=\frac{I_{x}-I_{-x}}{I_{0}}, M_{d d r}=\frac{\left(I_{x}-I_{-x}\right)-\left(I_{y}-I_{-y}\right)}{I_{0}}
$$

$I_{x}$ is the correlator output located $x$ chip away from the prompt correlator

$I_{0}$ is the prompt correlator output

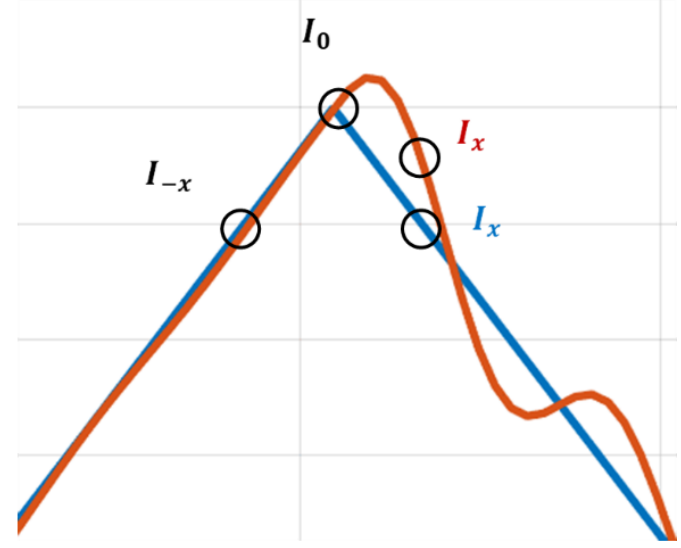

Figure 2. Nominal and distorted correlator outputs on the correlation function

In this SQM2b, it is assumed that all possible simple ratio, symmetric difference ratio and double difference ratio metrics based on all available correlator outputs are computed. For instance, assuming that there are 5 pairs of correlators available for the SQM, the number of metrics will be: 10 single ratio metrics +5 difference ratio metrics +5 sum ratio metrics $=20$ metrics.

Once the SQM2b algorithm computes all the metrics based on the chosen correlator outputs, the next step is to evaluate the detector value associated to each type of combination $\operatorname{Det}_{M}$ and compare it to each associated threshold. The Detector $\operatorname{Det}_{M}$ is the difference between the metric $M$ computed on the reference station signal and the metric $M^{\text {nominal }}$ computed in nominal conditions. Then the threshold test is evaluated as follow:

$$
\text { Test }_{M}=\frac{\text { Det }_{M}}{\text { threshold }_{M}}=\frac{M-M^{\text {nominal }}}{\text { threshold }_{M}}
$$

where $M$ stands for the metric output used to compute the detector value

Finally, the SQM2b monitor will decide that an abnormal distortion is present if any one of the detectors associated to each metric crosses the threshold:

\section{Threshold derivation}

$$
S Q M=\left\{\begin{array}{l}
1 \text { if } \exists M / \text { Test }_{M} \geq 1 \\
0 \text { if } \forall M \text { Test }_{M}<1
\end{array}\right.
$$

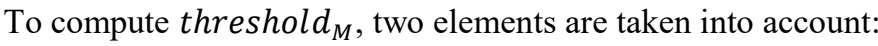

- integrity and continuity requirements relative to the required performance at the SBAS monitor level

- the fault free distribution of the used metric

Although, in reality the detection test is based on the use of a detection threshold, for simulation purposes it is better to compare the detector value (without noise) to the Minimum Detectable Error (MDE). Indeed, assuming that the detector has a Gaussian distribution, this comparison allows to assess if the detector meets both the allocated false alarm probability $P_{f a, m o n}$ and missed detection probability $P_{m d, m o n}$ associated to the monitor (for each distortion). In the next paragraph, the definition of $P_{m d, m o n}$ and of $P_{f a, m o n}$ is given. In this frame, the considered definition of MDE here is:

$$
M D E_{M}(\text { distortion })=\left(k_{m d}(\text { distortion })+k_{f a}\right) \sigma_{M}
$$


where

- $\sigma_{M}$ is the standard deviation of metric $M$ (after fusion of the observations from 4 stations and the metric smoothing)

- $\quad K_{f a}$ and $K_{m d}$ are respectively the fault-free detection and the missed detection multipliers associated to the detector $P_{f a}$ and $P_{m d}$ assuming a Gaussian distribution of the detector.

From a pure performance point of view, the SQM monitor will thus be performing in an appropriate way if:

$$
\forall \text { distortion } i, M a x_{-} D i f f_{-} \text {Bias }_{\text {risen/rising }}^{i}>M E R R \text { and } \exists M \text { such that } \frac{\operatorname{Det}_{M}(i)}{M D E_{M}(i)}>1
$$

Derivation of $P_{f a}$ and $P_{m d}$ for the EWF Monitor

The global false alarm and missed detection probabilities, allocated to EWF monitoring, need to be adequately mapped to each monitor. To do so, the SBAS architecture and the used monitors are analyzed.

\section{Derivation of $P_{f a}$ for the EWF Monitor}

First the SBAS architecture for EWF monitoring is assumed to be based on a centralized data fusion. This means that the EWF detection decision will be taken at the Central Processing Facility (CPF) level based on the data (observations or combination of observations) provided by the reference stations. A minimum number of four reference stations monitoring the satellite is considered. This means that the global false alarm and missed detection probabilities allocated to EWF monitoring are directly allocated to the CPF EWF monitor, which is composed of:

- the SQM detector includes several metrics based on correlators outputs measurements.

- CCI detector as an additional monitor (with the SQM metrics) on the absolute reference bias and only useful for the Risen satellite Scenario.

The requirement regarding the EWF monitor states a global $P_{f a}$ of $1.5 .10^{-7}$ per test. Assuming that all monitors are independent, the probability of false alarm for each monitor is:

$$
P_{f a, \text { SQMmon }}=\frac{P_{f a}}{L}
$$

where $L$ is the number of detectors constitutive of the monitor (including the CCI). This is a worst case because this minimizes the false alarm probability per detector and thus maximizes the detection threshold.

\section{Derivation of the $P_{m d}$ for generic fault monitor}

Regarding the $P_{m d}$ for any detector, as stated in [2], the actual required $P_{m d}$ will depend upon the differential range bias $E$ induced by a system fault in order to comply with the probability of integrity failure allocated to the fault $P_{I F}$. If the differential range bias is modelled as a random Gaussian variable, the probability that the induced bias $E$ exceeds the protection level is defined as:

$$
P_{P l}=Q\left(\frac{K_{f f d} \times \sigma_{f f, \min }-E}{\sigma_{a c c, f f}}\right)+Q\left(\frac{K_{f f d} \times \sigma_{f f, \min }+E}{\sigma_{a c c, f f}}\right)
$$

where $Q(x)$ is the probability that a normalized centered Gaussian variable is greater than $x$

- $K_{f f d}$, the multiplier corresponding to the computation of the PL is 5.33 (corresponding to an integrity risk of 1e-7)

- $\sigma_{a c c, f f}$ is the fault free range accuracy taking into account clock and orbit error for DF. It is used to define the pseudorange bias distribution.

- $\sigma_{f f, \min }$, the standard deviation of the corrected measurements used to compute the PL threshold. In the present case, it is important to take a lower bound in order to have margins:

$$
\sigma_{f f, \min }=\sqrt{\sigma_{D F C}^{2}+\sigma_{\text {Tropo }}^{2}+\sigma_{M P, D F}^{2}+\sigma_{\text {Noise }, D F}^{2}+\sigma_{\text {IonoRes }, D F}^{2}}
$$

- $\sigma_{D F C}^{2}$ is the model variance for the residual error that can be lower bounded by $\sigma_{D F R E}^{2}$.

- $\sigma_{\text {Tropo }}^{2}$ is the model variance for the residual tropospheric error.

- $\sigma_{M P, D F}^{2}$ is the model variance for the multipath error (after smoothing).

- $\sigma_{\text {Noise }, D F}^{2}$ is the model variance for the noise error (after smoothing) assumed to be equal to 0 . 
- $\quad \sigma_{\text {IonoRes,DF }}^{2}$ is the model variance for the residual ionospheric error (after smoothing) assumed to be equal to 0 .

In order to protect the SBAS user against any integrity failure caused by the considered failure, the $P_{m d}$ of the associated monitor has to satisfy the following inequality:

$$
\frac{P_{I F}}{P_{f} \times P_{m d}} \leq P_{P L}
$$

Hence, the required missed detection probability $P_{m d}$ for the fault inducing the differential bias $E$ is then the $P_{m d}$ corresponding to the limit value achieving the equality in the previous equation and it can be computed as:

Where

$$
P_{m d}=\frac{P_{I F}}{P_{f} \times\left(Q\left(\frac{K_{f f d} \times \sigma_{f f, \min }-E}{\sigma_{a c c, f f}}\right)+Q\left(\frac{K_{f f d} \times \sigma_{f f, \min }+E}{\sigma_{a c c, f f}}\right)\right)}
$$

- $\quad P_{I F}$ is the probability of integrity failure allocated to the considered fault

- $\quad P_{f}$ is the a priori fault probability

\section{Derivation of the $P_{m d}$ for the EWF monitor}

Let us apply the generic $P_{m d}$ formula to compute the allocated $P_{m d}$ to both SQM and CCI metrics

Let us denote $P_{m d, S Q M}^{R i s i n g, i}$ and $P_{m d, S Q M}^{\text {Risen, } i}$ the missed detection probability that the SQM should fulfill for distortion $i$ in the rising and risen case respectively. Let us assume as an example that we are looking at the detection of a specific distortion and that this distortion creates a steady-state bias $E_{k, m}^{i}\left(t_{s s}\right)$ that is distinct for all possible user of the user design space.

- In the rising scenario, the SQM monitor will protect adequately any user if the SQM monitor has a $P_{m d}$ that is lower than all $P_{m d, S Q M}^{i}\left(E_{k, m}^{i}\left(t_{s s}\right)\right)$ computed for all differential bias in $E_{k, m}^{i}\left(t_{s s}\right)$ associated to all user types of the user space. This means that

- Each user type will have its own $P_{m d, S Q M}^{i}\left(E_{k, m}^{i}\left(t_{s s}\right)\right)$ requirement corresponding to the differential bias $E_{k, m}^{i}\left(t_{s s}\right)$ created by the considered distortion.

- Since the SQM monitor has to protect all user types, the most demanding Pmd over all user types for a specific distortion should be adopted as the SQM monitor Pmd requirement:

$$
P_{m d, S Q M}^{\text {Rising } i}=\min _{k, m \in \text { User Space }}\left(P_{m d, S Q M}^{i}\left(E_{k, m}^{i}\left(t_{s s}\right)\right)\right)
$$

- In the risen scenario, the same mechanism is at play to select the required $P_{m d, S Q M}^{R i s e n, i}$ per distortion. The difference is that now, the differential range bias considered is a time-varying differential bias $E_{k, m}^{i}(\mathrm{t})$. This results in a time varying $P_{m d, S Q M}^{i}\left(E_{k, m}^{i}(\mathrm{t})\right)$. The worst $P_{m d}$ during the transient period induced by the EWF occurrence is evaluated as

$$
P_{m d, S Q M}^{\text {Risen, }, k, m}=\min _{t}\left(P_{m d, S Q M}^{, i}\left(E_{k, m}^{i}(\mathrm{t})\right)\right)
$$

Then, the required $P_{m d}$ for a given distortion is the most demanding constraint on the SQM monitor among the user space. The corresponding $P_{m d, S Q M}^{R i s e n}, i$ has the following expression:

$$
P_{m d, S Q M}^{\text {Risen }, i}=\min _{k, m \in \text { User Space }}\left(P_{m d, S Q M}^{\text {Risen }, i, k, m}\right)
$$

In order to check if the CCI monitor has already detected the considered fault, it is tested with the required $P_{m d, S Q M}^{\text {Risen,i }}$ (as Test $\left.t_{C C I}^{i}=\frac{\text { bias }_{C C I}^{E W F i}-\text { bias }_{C I}^{\text {nominal }}}{\left(k_{m d^{i}}^{i} k_{f a}\right) \sigma_{C C I}}\right)$. If the CCI test is successful (Test $\left.t_{C C I}^{i} \geq 1\right)$, the CCI monitor is compliant with the integrity requirement, and no requirement on the SQM monitor has to be made. Then $P_{m d, S Q M}^{R i s e n}, i$ is set to 1 .

At the end, the targeted $P_{m d, S Q M}^{i}$ for the distortion $i$ will thus be:

$$
P_{m d, S Q M}^{i}=\min \left(P_{m d, S Q M}^{\text {Rising }, i}, P_{m d, S Q M}^{\text {Risen }, i}\right)
$$




\section{EVALUATION OF THE SQM2B AND THE CCI DETECTABILITY}

It is here important to remind the choice for the simulation parameters given in Table 3. The SQM2b and CCI compliance with the integrity requirements for TM-A, TM-B and TM-C for Galileo E1c and E5a is presented in this section.

In the risen scenario the time variant differential bias and SQM monitor obtained as an output of the smoothing filter are used to evaluate the worst transient differential bias. The considered periods for the smoothing filter in the risen case are:

- $100 \mathrm{~s}$ for the airborne receiver (applied to the absolute bias)

- $600 \mathrm{~s}$ for the ground receiver (applied to the reference bias)

- $25 \mathrm{~s}$ for the SQM filter

Table 3 - Assumptions used to generate the Galileo E1c and E5a EWF Threat Space

\begin{tabular}{|c|c|c|c|c|}
\hline & \multicolumn{2}{|c|}{ Galileo E1c signal (CBOC(6.1)) } & \multicolumn{2}{|c|}{ Galileo E5a and GPS L5 signal BPSK(10)) } \\
\hline & reference & user & reference & User \\
\hline $\begin{array}{l}\text { Tracking } \\
\text { technique }\end{array}$ & $\begin{array}{l}\text { EML }(\text { BOC }(1.1) \\
\text { local replica) }\end{array}$ & $\begin{array}{l}\text { EML (BOC(1.1) } \\
\text { local replica) }\end{array}$ & $\begin{array}{l}\text { EML (BPSK(10) } \\
\text { local replica) }\end{array}$ & $\begin{array}{l}\text { EML }(\text { BPSK }(10) \\
\text { local replica) }\end{array}$ \\
\hline $\begin{array}{l}\text { Correlator } \\
\text { spacing }\end{array}$ & \multicolumn{2}{|c|}{$\begin{array}{c}0.08,0.1 \text { and } \\
0.12 \text { chip }\end{array}$} & \multicolumn{2}{|c|}{$0.9,1$ and 1.1 chip } \\
\hline $\begin{array}{l}\text { Pre-correlation } \\
\text { bandwidth } \\
\text { (double-sided) }\end{array}$ & $24 \mathrm{MHz}$ & $\begin{array}{l}12,14,16,18,20 \\
22,24 \mathrm{MHz}\end{array}$ & $24 \mathrm{MHz}$ & $\begin{array}{l}12,14,16,18,20 \\
22,24 \mathrm{MHz}\end{array}$ \\
\hline \multirow[t]{2}{*}{$\begin{array}{l}\text { Equivalent } \\
\text { reception filter }\end{array}$} & $\begin{array}{l}\text { one filter is tested } \\
\text { (6th-order } \\
\text { Butterworth) to } \\
\text { estimate the } \\
\text { reference error as } \\
\text { the minimum on all } \\
\text { the correlator } \\
\text { spacing values }\end{array}$ & $\begin{array}{l}4 \text { filters are tested } \\
\text { (6th-order } \\
\text { Butterworth, 0- } \\
\text { group delay } \\
\text { resonator, } 150 \mathrm{~ns} \\
\text { differential group } \\
\text { delay resonator, } 150 \\
\text { ns differential group } \\
\text { delay 6th-order } \\
\text { Butterworth) }\end{array}$ & $\begin{array}{l}\text { one filter is tested } \\
\text { (6th-order } \\
\text { Butterworth) to } \\
\text { estimate the } \\
\text { reference error as the } \\
\text { minimum on all the } \\
\text { correlator spacing } \\
\text { values }\end{array}$ & $\begin{array}{l}4 \text { filters are tested } \\
\text { (6th-order } \\
\text { Butterworth, 0- } \\
\text { group delay } \\
\text { resonator, } 150 \mathrm{~ns} \\
\text { differential group } \\
\text { delay resonator, } 150 \\
\text { ns differential group } \\
\text { delay 6th-order } \\
\text { Butterworth) }\end{array}$ \\
\hline & \multicolumn{4}{|c|}{$\begin{array}{c}\text { Differential tracking error is estimated as the maximum on all combinations of user and } \\
\text { reference configurations. }\end{array}$} \\
\hline
\end{tabular}

\section{Results for Galileo E1c}

In this section, the obtained detectability performance when applying the SQM2b and the CCI taking into account the integrity requirement presented priviousely is shown for both scenario Risen and Rising.

The three presented SQM2b metrics $\left(M_{s r}, M_{s d r}\right.$ and $\left.M_{d d r}\right)$ can be written as:

$$
M=S_{M}^{T} I_{x}
$$

Where $I_{x}$ is a vector of normalized correlator outputs and $S_{M}$ is a vector of coeffcients $S_{m}^{i} \in\left\{-\frac{1}{2}, 0, \frac{1}{2}\right\}$.

The standard deviation of each metric $\mathrm{M}$ is computed using the covariance matrix of the chosen correlator outputs $\mathrm{Cov}$ as:

$$
\sigma_{M}=\sqrt{S_{M}^{T} \operatorname{Cov} S_{M}}
$$

In this study, the covariance matrix $\operatorname{Cov}$ is evaluated based on a Monte Carlo simulation of the correlation operation taking into account only thermal Gaussian noise at a $\mathrm{C} / \mathrm{N} 0$ of $30 \mathrm{dBHz}$. The multipath effect on the noise level is not considered here. The covariance matrix is also taking advantage of the applied smoothing filter with a period of $25 \mathrm{~s}$ and the reduction factor of 2 since in the considered centralized architecture, the SQM data of at least 4 reference stations are combined in the SBAS ground segment. 
The considered noise level here is taking large margin since it considers that the four reference stations monitoring the satellite are all at the lowest elevation with a very low $\mathrm{C} / \mathrm{N} 0$ level. This margin should cover the multipath effect on the noise which is not added here.

The proposed SQM2b for Galileo E1c uses 13 correlator outputs at $+/-[0.02 ; 0.03 ; 0.04 ; 0.06 ; 0.08 ; 0.1]$ chips. Note that this SQM is considered as a simple non-optimized algorithm that can be easily implemented in GBAS or SBAS.

The standard deviation of the CCI monitor $\sigma_{C C I}$ (used to evaluate the threshold $d_{C C I}$ ) is set to $12 \mathrm{~cm}$ for Galileo E1c signal after applying the same smoothing period and reduction factor of the SQM monitors. This value is proposed here based on the result of real data assessment for several formula of $\mathrm{CCI}$ (or $\mathrm{CCD}$ ) monitors as already mentioned.

\section{$\underline{\text { Assessment of detectability for TM-A }}$}

The covered TM-A area by the CCI and the SQM2b are given in Figure 3 with the induced maximum transient and steady state differential bias observed respectively in the risen (right) and rising (left) case for TM-A distortions. The green areas represent the $\Delta$ values where the SQM2b and the CCI tests exceeds the threshold and so detect the fault occurrence. Based on the results of this figure, the efficiency of the applied monitors to detect hazardous TM-A cases in both scenarios is assessed:

- In the rising scenario, the maximum steady state differential bias (black line in the left figure) is measured and only the SQM2b test is applied to define the covered area. All TM-A distortions with a steady state differential bias exceeding the MERR of $1 \mathrm{~m}$ for Galileo E1c needs to be covered by the SQM2b metrics. Since all distortions with differential bias above the red line of the MERR limit is included in the green area, it is clear that all hazardous TM-A in the rising scenario are covered by the SQM2b.

- In the risen case, the maximum transient differential bias (blue line in the figure on the right) is assumed to be the worst bias that can be observed. The green areas represent the $\Delta$ cases covered by the CCI monitor. Figure 3 shows that all hazardous distortions with maximum transient differential bias larger than MERR of $1 \mathrm{~m}$ (above the red dashed line) are covered by the CCI test (since they are included in the green area). Note that the SQM2b is not necessary in the risen case.
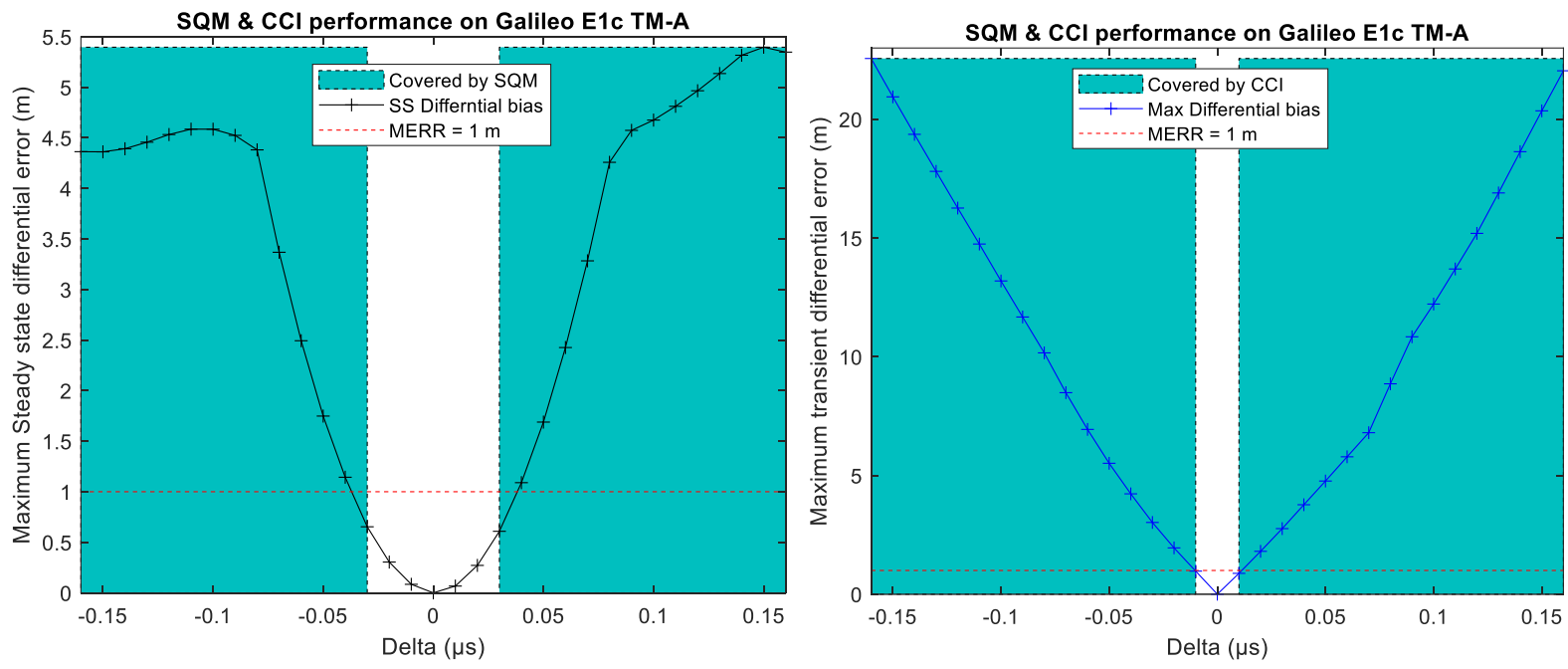

Figure 3. SQM2b and CCI test results with Maximum differential bias in the rising (right) and risen scenario (left) for TM-A on Galileo E1c

Hence, all the hazardous TM-A cases (included in the tested TS: $\Delta \in[-0.16 ; 0.16] \mu \mathrm{s}$ ) in both risen and rising scenario are covered by the CCI and the proposed SQM2b. The detectability of the CCI and the SQM2b has not been tested outside a $\Delta \in$ $[-0.16 ; 0.16] \mu \mathrm{s}$.

\section{$\underline{\text { Assessment of detectability for TM-B }}$}


Figure 4 shows the obtained performance with the proposed SQM2b and the CCI for TM-B distortions with the $1 \mathrm{~m}$ iso-contour representing the MERR limit for Galileo E1c signal on the maximum steady sate and transient differential bias. The analysis of these results for risen and rising scenarios allows us to conclude on the covered areas by the CCI and the SQM2b for TM-B distortions.

- The figure on the left shows the results for rising scenario. The green area represents the TM-B cases detected by the SQM2b. This area includes all Hazardous TM-B distortions in the Rising scenario which have a steady state differential bias exceeding the MERR of $1 \mathrm{~m}$ (located below the red contour at $1 \mathrm{~m}$ ). Hence the proposed SQM2b is able to cover all TM-B cases exceeding the MERR for Galileo E1c.

- In the Risen case, the CCI and the SQM2b are applied as shown in the figure on the right. The iso-contour at $1 \mathrm{~m}$ (in red) define the area of TM-B with maximum transient differential bias exceeding the MERR limit. It appears that the whole area is covered by the combination of the CCI and the SQM2b.

Then all TM-B distortions that induce a differential bias larger than the MERR limit in the risen or the rising scenario are detected by the CCI and SQM2b monitors.
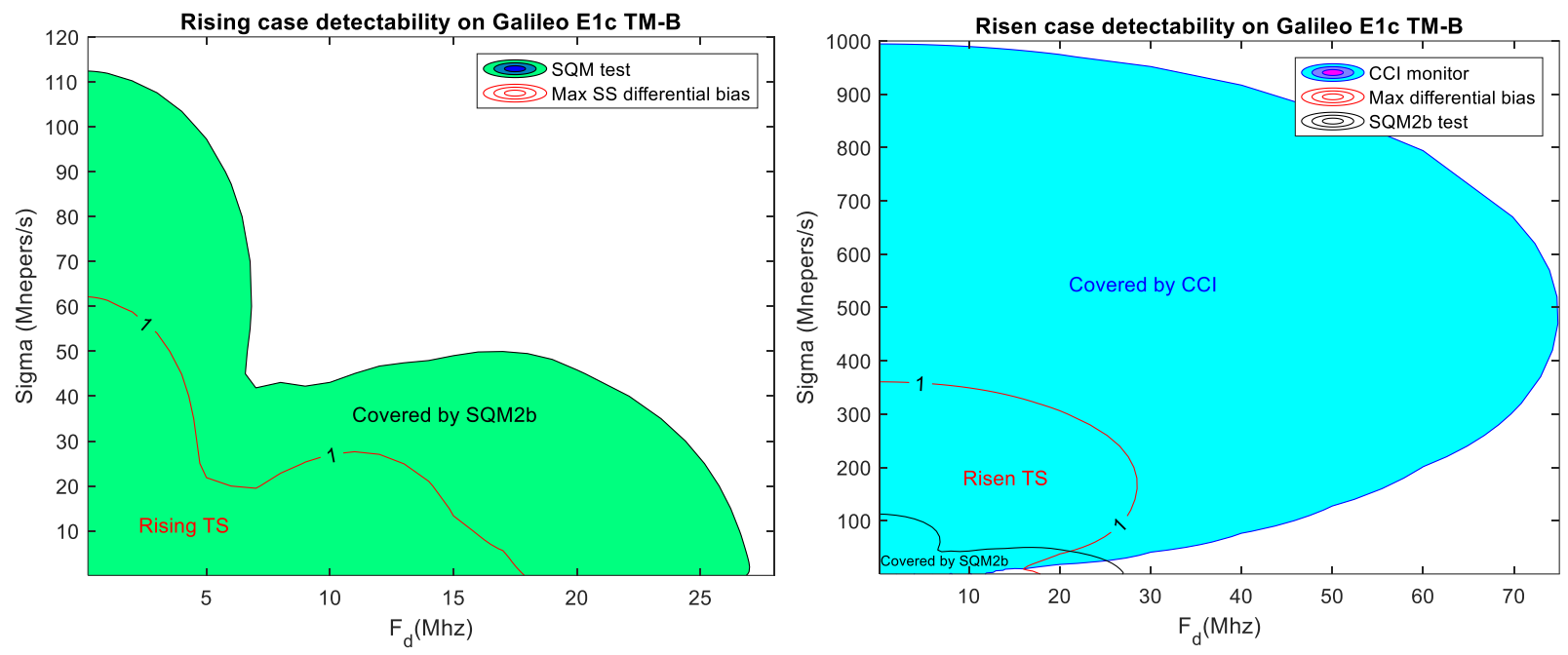

Figure 4. SQM2b and CCl test results for Risen (right) and Rising (left) scenarios applied to the TM-B for Galileo E1c

\section{$\underline{\text { Assessment of detectability for TM-C }}$}

Figure 5 shows the observed differential bias in the risen and rising scenarios as a function of the highest metric test among the applied monitors for the TM-C distortions given in Table 2. Each blue circle represents a TM-C distortion defined by the parameters $\left(\Delta, \sigma\right.$ and $\left.f_{d}\right)$. The figure on the left presents the results in the rising case by giving the maximum steady state differential bias as a function of the SQM2b test metrics. In this case the $P_{m d}$ requirement evaluated for each distortion as presented previously is computed only for the rising scenario. The figure on the right represents the maximum transient differential bias as a function of the highest SQM2b and CCI test. These figures show the obtained performance while considering the $P_{m d}$ requirement for each scenario separately. It appears that in both scenarios, all distortions with differential bias exceeding the MERR limit (above the red line) are located at the right side of the black line representing the limit where metric test is equal to 1 . Thus, they are all detected by the proposed SQM2b metrics and CCI. 

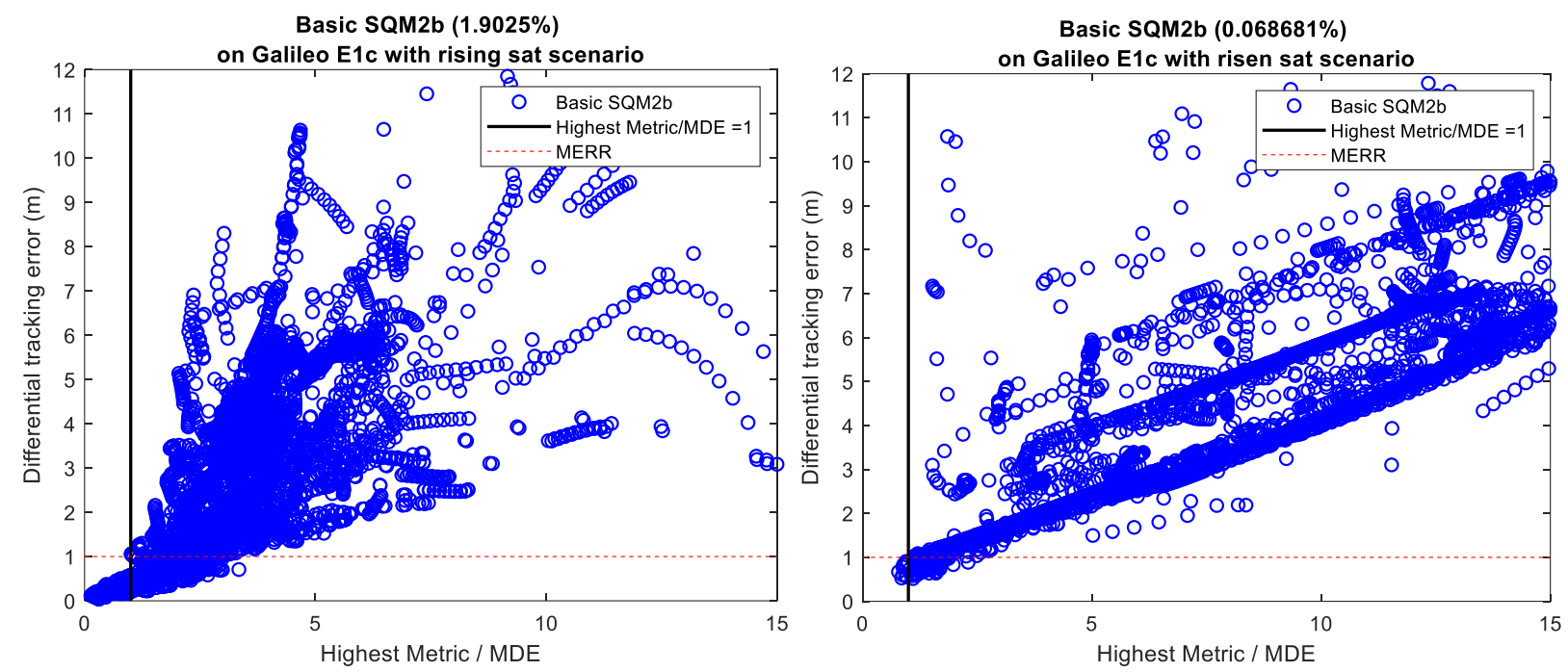

Figure 5. Results of SQM $2 b$ with correlator at [ 0.020 .030 .040 .060 .080 .1 ] chip and $\mathrm{CCl}$ (with $\sigma_{C C I}=0.12 \mathrm{~cm}$ ) test performance in the Rising (left) and Risen scenario (right) applied to the extended TM-C TS for Galileo E1C

Note that the presented CCI and SQM2b performances are valid only for the tested TM-C TS (given in Table 2).

\section{Results for Galileo E5a}

The same analysis as the one used for Galileo E1c is applied here for Galileo E5a to determine the covered area of TMA, TMB threat space in the rising and risen scenarios and validate this performance for the TM-C.

The proposed SQM for Galileo E5a is an SQM2b with 11 correlator outputs located at $[0.2 ; 0.4 ; 0.6 ; 0.8 ; 1]$ chip. The standard deviation for each metric is evaluated based on the same theoretical model used for Galileo E1c. The CCI standard deviation is set to $30 \mathrm{~cm}$.

\section{$\underline{\text { Assessment of detectability for TM-A }}$}

The CCI and SQM2b capability of detecting hazardous TM-A cases in the risen and the rising cases is assessed based on the analysis of the results shown in Figure 6. As for Galileo E1c, the covered areas by the CCI and the SQM2b, the maximum steady state and transient differential bias and the limit of the MERR are given. The analysis of the monitor test results in each scenario shows that:

- In the rising scenario, all TMA distortions with a steady state differential bias (black line in the left figure) exceeding the MERR is included in the SQM2b covered area. So, the proposed SQM2b detects all hazardous TM-A cases.

- In the risen case, all distortions with a maximum transient differential bias (blue line in the figure on the right) larger than the MERR of $2 \mathrm{~m}$ are located inside the green defined covered by the CCI monitor. So, all hazardous TM-A cases in the risen scenario are detected by the CCI. Note that the SQM2b is not necessary in the risen case. 

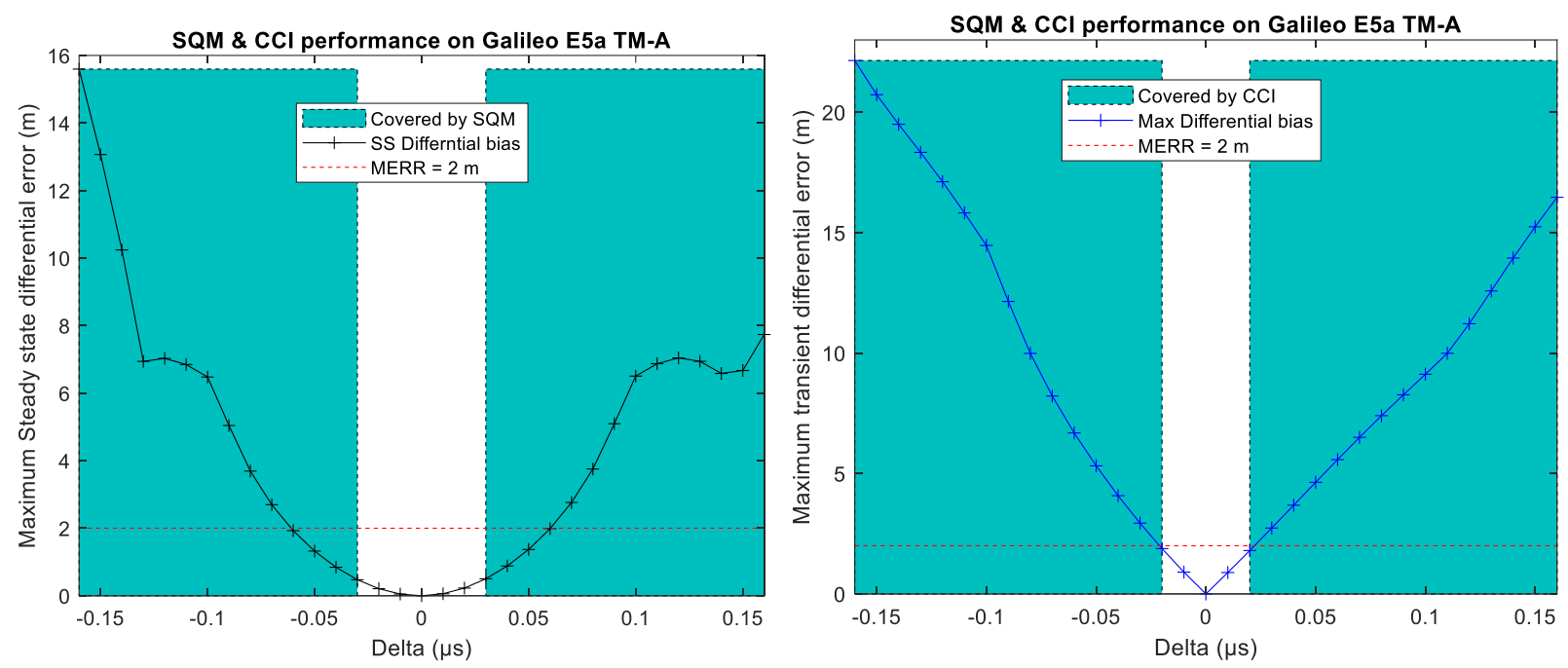

Figure 6. SQM2b and $\mathrm{CCl}$ test results with Maximum differential bias in the rising (right) and risen scenario (left) for TM-A on Galileo E5a

Then, all hazardous TM-A in the tested TS are covered by the CCI and the SQM2b in the risen and rising scenario. The detectability of the CCI and the SQM2b has not been tested outside a $\Delta \in[-0.16 ; 0.16] \mu$ s.

\section{$\underline{\text { Assessment of detectability for TM-B }}$}

Figure 7 shows the SQM2b and CCI performances and the TM-B area defined by the iso-contour at MERR of $2 \mathrm{~m}$. The analysis of these results in both risen (figure on the right) and rising (figure on the left) scenarios confirms that the proposed SQM2b and CCI are sufficient to cover all hazardous cases:

$\circ \quad$ In the rising scenario, SQM2b covers the area below the limit of steady state differential bias larger than $2 \mathrm{~m}$ except for distortions with $\sigma$ lower than 20 Mnepers/s and $f_{d}$ lower than $4 \mathrm{MHz}$. However, in this area all distortions are characterized by a correlation loss larger than the limit of $15 \mathrm{~dB}$ (which is shown in pink in Figure 7). It is assumed that airborne and ground receivers experiencing a correlation loss larger than $15 \mathrm{~dB}$ are likely to lose the signal tracking. It is so expected that above $15 \mathrm{~dB}$ of signal power degradation, the satellite will not be monitored by SBAS. This means that the area delimited by the iso-contour at $15 \mathrm{~dB}$ of correlation loss can be removed from the set of the Hazardous TM-B distortions. So, all remaining cases are covered by the proposed SQM2b in the rising case.

- In the risen scenario, the figure on the left show that all hazardous distortions with maximum transient differential bias larger than the MERR of $2 \mathrm{~m}$ are covered by the combination of the CCI and the proposed SQM2b. As mentioned for the rising case, the area of distortions characterized by a correlation loss exceeding the limit of $15 \mathrm{~dB}$ are removed from the TM-B TS. 

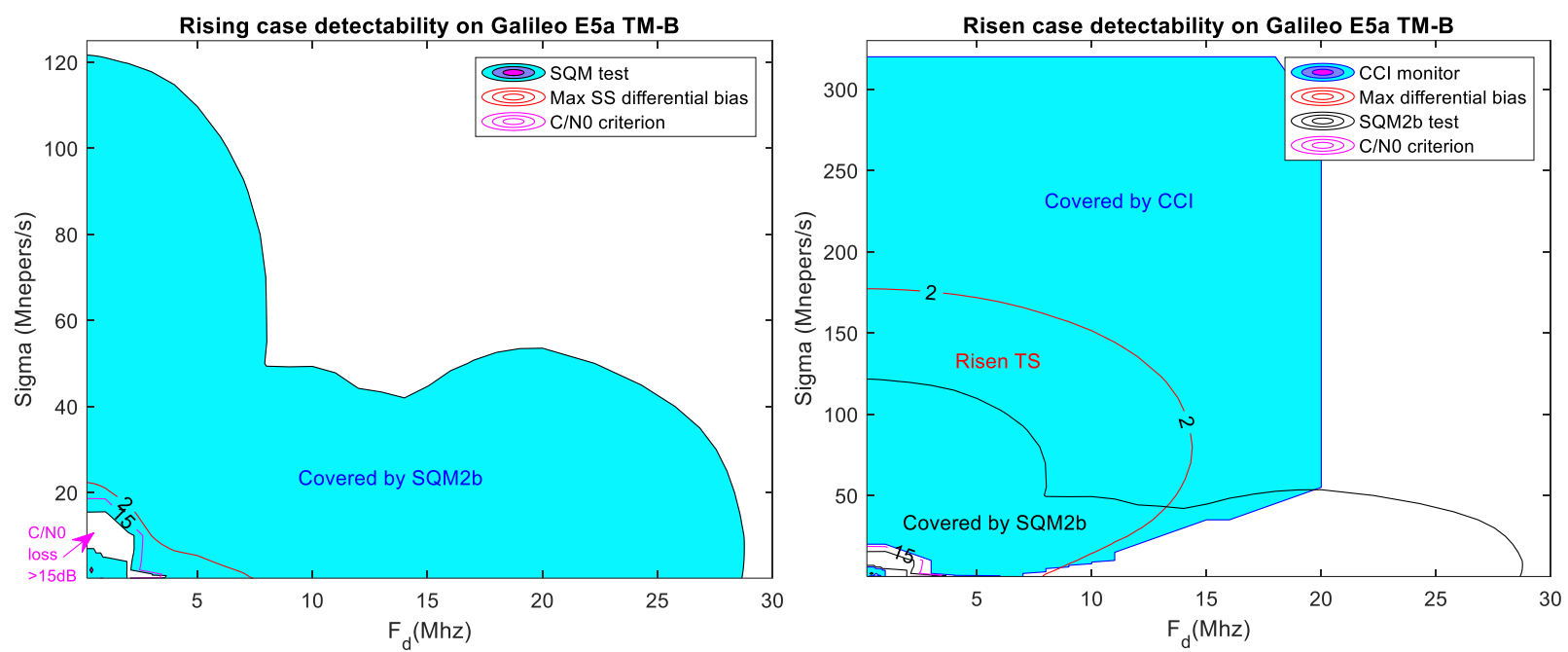

Figure 7. SQM $2 b$ and $C C I$ test results for Risen (right) and Rising (left) scenarios applied to the TM-B for Galileo E5a

\section{Assessment of detectability for TM-C}

The obtained results for the tested TS in the risen and the rising case are shown in Figure 8. It appears that the hazardous TM-C cases with differential bias exceeding the MERR of $2 \mathrm{~m}$ in the tested TS are detected by the proposed SQM2b and CCI while respecting the $P_{f a}$ and $P_{m d}$ requirement in the risen and rising cases.
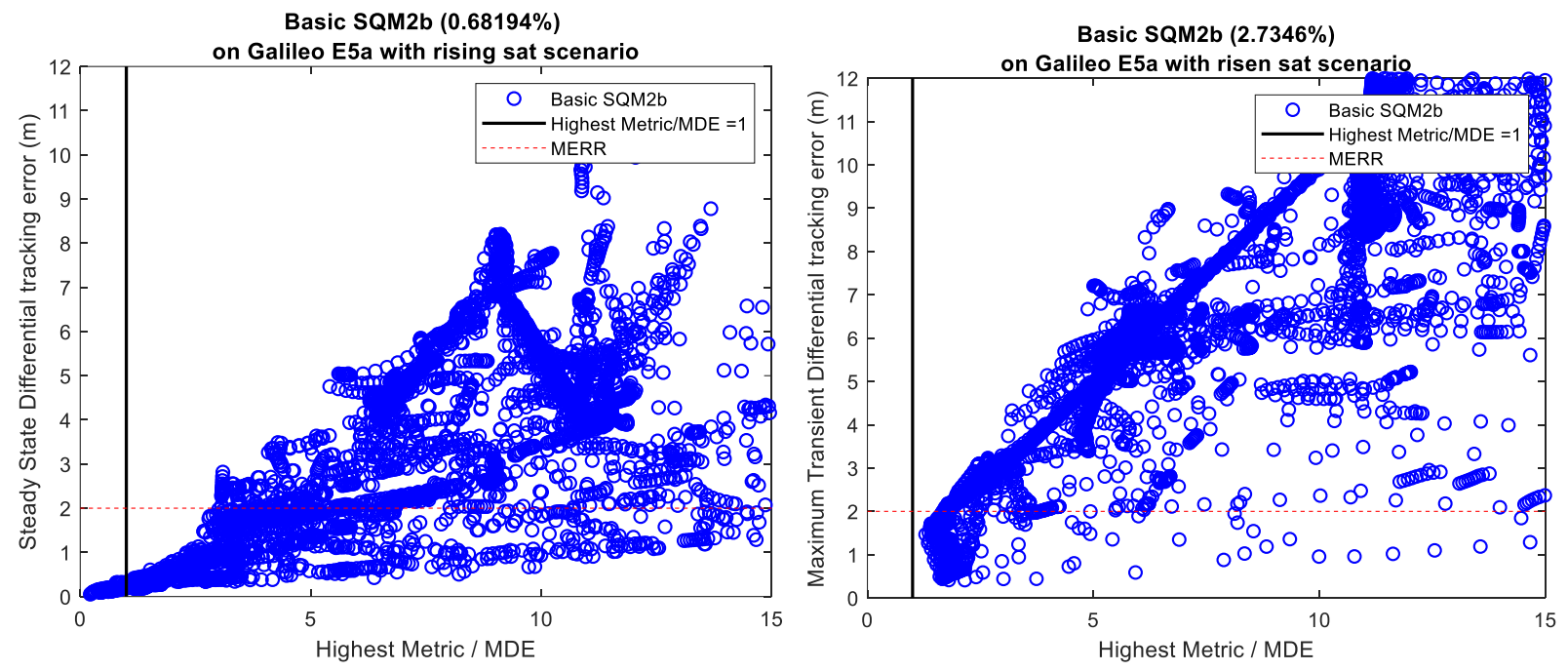

Figure 8. Results of SQM $2 b$ and $\mathrm{CCl}$ test performance in the Risen (right) and Rising (left) scenarios applied to the TM-C for Galileo E5a.

Note that, as for Galileo E1c, the detectability of the CCI and the SQM2b has not been tested outside a $\Delta \in[-0.16 ; 0.16] \mu \mathrm{s}$.

\section{CONCLUSION}

In this paper, an SQM2b based on the use of 13 and 11 correlators outputs for Galileo E1c and E5a respectively is proposed to detect hazardous EWF distortions in the tested TS. In addition, in the risen scenario, CCI monitor with standard deviation of $12 \mathrm{~cm}$ on Galileo E1c and $30 \mathrm{~cm}$ on Galileo E5a is applied on the reference bias measurement. The compliance of this SQM2b and CCI monitors with the SBAS requirement in term of $P_{f a}$ and $P_{m d}$ when detecting EWF threats is assessed. The standard deviation of the 
SQM2b metrics takes into account only white Gaussian noise effect at a low C/N0 of $30 \mathrm{dBHz}$ for all reference stations monitoring the considered satellite.

The presented performances of these monitors show that they cover all hazardous EWF cases in the TM-A, TM-B and TM-C in the tested TS for Galileo signals. The tested TS includes the Galileo TS proposed in ICAO SARPs [8].

Note that all the presented performances are based on a large set of assumptions used to define the DFMC SBAS context (both integrity requirements and system architecture and performance), such as:

- All measurements at ground station are made with a $\mathrm{C} / \mathrm{N} 0$ equal to $30 \mathrm{dBHz}$

- The detection metrics are averaged only over 4 stations

- MERR safety margin for E1 and E5a cases

If these assumptions are modified with different constraints, the performance presented for the SQM2b and the CCI monitor need to be updated.

In future work, it is expected to use a less demanding CCI monitor with an optimized SQM giving better performance on the tested TS. There is also a need to include the multipath effect at the reference station level in the estimation of the monitors covariance to validate the obtained performance with more realistic data.

\section{ACKNOWLEDGMENTS}

Dr. Olivier JULIEN's work contributing to this paper was exclusively performed when he was ENAC employee.

\section{REFERENCES}

[1] ICAO. Annex 10 - Aeronautical Telecommunications, Volume 1 - Radio Navigation Aids [Internet]. Sixth Edition. Vol. 1. 2006.

[2] Rife J, Phelts RE. Formulation of a time-varying maximum allowable error for ground-based augmentation systems. IEEE Transactions on Aerospace and Electronic Systems. 2008 Apr;44(2):548-60.

[3] Shallberg KW, Ericson SD, Associates Z, Phelts E, Walter T, Kovach K, et al. Catalog and Description of GPS and WAAS L1 C/A Signal Deformation Events. In Monterey, California; 2017.

[4] Shloss P, Phelts RE, Walter T, Enge P. A Simple Method of Signal Quality Monitoring for WAAS LNAV/VNAV. In: 15th International Technical Meeting of The Satellite Division of the Institute of Navigation [Internet]. Portland, Oregon; 2002.

[5] Jiang Y, Milner C, Macabiau C. Code carrier divergence monitoring for dual-frequency GBAS. GPS Solutions [Internet]. 2016 Sep 28 [cited 2017 Mar 9]; Available from: http://link.springer.com/10.1007/s10291-016-0567-4

[6] Phelts E, Akos D, Enge P. Robust Signal Quality Monitoring and Detection of Evil Waveforms. In: 13th International Technical Meeting of The Satellite Division of the Institute of Navigation [Internet]. Salt Lake City, Utah; 2000 [cited 2016 Jun 6].

[7] Phelts E, Walter T, Enge P. Toward Real-Time SQM for WAAS: Improved Detection Techniques. In: 16th International Technical Meeting of The Satellite Division of the Institute of Navigation [Internet]. Portland, Oregon; 2003.

[8] Mabilleau M, Kawak B, Cordero Limon M, Wallner S, Spinelli S. Proposal for Galileo Evil Wave Form Threat Space. In Montreal; 2018. 